\title{
Communication: Direct tests of single-parameter aging
}

Hecksher, Tina; Olsen, Niels Boye; Dyre, Jeppe C.

Published in:

Journal of Chemical Physics

DOI:

$10.1063 / 1.4923000$

Publication date:

2015

Document Version

Publisher's PDF, also known as Version of record

Citation for published version (APA):

Hecksher, T., Olsen, N. B., \& Dyre, J. C. (2015). Communication: Direct tests of single-parameter aging. Journal of Chemical Physics, 142, [241103]. https://doi.org/10.1063/1.4923000

\section{General rights}

Copyright and moral rights for the publications made accessible in the public portal are retained by the authors and/or other copyright owners and it is a condition of accessing publications that users recognise and abide by the legal requirements associated with these rights.

- Users may download and print one copy of any publication from the public portal for the purpose of private study or research.

- You may not further distribute the material or use it for any profit-making activity or commercial gain.

- You may freely distribute the URL identifying the publication in the public portal.

Take down policy

If you believe that this document breaches copyright please contact rucforsk@kb.dk providing details, and we will remove access to the work immediately and investigate your claim. 


\section{Communication: Direct tests of single-parameter aging}

Tina Hecksher, Niels Boye Olsen, and Jeppe C. Dyre ${ }^{a}$

DNRF Center "Glass and Time," IMFUFA, Department of Sciences, Roskilde University, P.O. Box 260, DK-4000 Roskilde, Denmark

(Received 23 May 2015; accepted 15 June 2015; published online 30 June 2015)

This paper presents accurate data for the physical aging of organic glasses just below the glass transition probed by monitoring the following quantities after temperature up and down jumps: the shear-mechanical resonance frequency $(\sim 360 \mathrm{kHz})$, the dielectric loss at $1 \mathrm{~Hz}$, the real part of the dielectric constant at $10 \mathrm{kHz}$, and the loss-peak frequency of the dielectric beta process $(\sim 10 \mathrm{kHz})$. The setup used allows for keeping temperature constant within $100 \mu \mathrm{K}$ and for thermal equilibration within a few seconds after a temperature jump. The data conform to a new simplified version of the classical Tool-Narayanaswamy aging formalism, which makes it possible to calculate one relaxation curve directly from another without any fitting to analytical functions. (C) 2015 AIP Publishing LLC. [http://dx.doi.org/10.1063/1.4923000]

Gradual changes of material properties are referred to as aging. These are often caused by slow chemical reactions, but in some cases they reflect the so-called physical aging, which results exclusively from changes in atomic or molecular positions. ${ }^{1-14}$ For applications, it is important to be able to predict how fast material properties change over time, as well as in production. ${ }^{15-20}$ For instance, the performance of a smartphone's display glass is governed by the volume relaxation taking place when the glass is cooled through the glass transition. $^{21}$

Physical aging has been studied in publications dealing with the aging of, e.g., oxide glasses, ${ }^{4-7}$ polymers, ${ }^{3,8,16,17,19,22}$ metallic glasses, ${ }^{23,24}$ spin glasses, ${ }^{25,26}$ relaxor ferroelectrics, ${ }^{27}$ and soft glassy materials like colloids and gels. ${ }^{28,29}$ Quantities probed to monitor aging are, e.g., density, ${ }^{3,30}$ enthalpy, ${ }^{4,5}$ Young's modulus, ${ }^{23}$ gas permeability, ${ }^{31}$ high-frequency mechanical moduli, ${ }^{32,33} \mathrm{dc}$ conductivity, ${ }^{8}$ frequency-dependent dielectric constant, ${ }^{34-37} \mathrm{X}$-ray photon correlation spectroscopyprobed structure, ${ }^{38}$ and non-linear dielectric susceptibility. ${ }^{39}$

Physical aging is generally nonexponential in time and nonlinear in temperature variation. The focus below is on the aging of glasses just below their glass transition temperature, which is characterized by self-retardation for temperature down jumps and self-acceleration for up jumps. ${ }^{10,19,40-42}$ The standard aging formalism is due to Narayanaswamy, an engineer at Ford Motor Company who back in 1970 needed a theory for predicting how the frozen-in stresses in a windshield depend on the glass' thermal history. The resulting so-called Tool-Narayanaswamy (TN) theory accounts for the nonexponential and nonlinear nature of aging, as well as the crossover (Kovacs) effect reflecting memory of the thermal history. ${ }^{10,43}$ The TN trick is to assume the existence of an "inner clock" that defines a so-called material time. ${ }^{44-47}$ This is like the proper-time concept of the theory of relativity giving the time measured on a clock traveling with the observer. During aging, the clock rate itself ages, which causes nonlinearity in

\footnotetext{
a)Electronic address: dyre@ ruc.dk
}

temperature variation. A crucial assumption of the TN theory is that the "fictive temperature" controls both the clock rate and the quantity being monitored. This single-parameter assumption is usually tested by fitting data to analytical functions; below, we develop a simplified TN theory that may be tested directly from data without any fitting.

This paper presents accurate aging data for organic glasses obtained by monitoring the following four quantities after temperature jumps: the high-frequency shear-mechanical resonance frequency, ${ }^{32}$ the low-frequency dielectric loss (data from Ref. 48), ${ }^{34,49}$ the high-frequency real part of the dielectric constant, ${ }^{49}$ and the dielectric loss-peak frequency of the beta process (data partly published in Ref. 50). The setup used is described in Refs. 51 and 52. It is based on a custommade cryostat capable of keeping temperature constant within $100 \mu \mathrm{K}$ for the first three quantities and within $1 \mathrm{mK}$ for the fourth. A Peltier element is used for the cryostat's inner temperature control, and the time constant for equilibration of the setup after a temperature jump is only $2 \mathrm{~s}$. The dielectric measurements were made with a homebuilt setup that uses a digital frequency generator below $100 \mathrm{~Hz}$ producing a sinusoidal signal with voltages reproducible within $10 \mathrm{ppm}$; at higher frequencies, a standard LCR meter is used. The mechanical resonance measurements were carried out using a one-disc version of our piezo-ceramic shear transducer. ${ }^{53}$ See the supplementary material for more details. ${ }^{54}$

The three liquids studied are tetramethyl-tetraphenyltrisiloxane (DC704), 5-polyphenyl-4-ether (5PPE), and tripropylene glycol (TPG). Examples of the measurements behind the aging analysis are given in Fig. 1 (for a more thorough discussion please refer to Ref. 54). Figure 2 shows how the monitored quantity $X(t)$ equilibrates upon temperature up and down jumps (black and light blue symbols). There is always a rapid change of $X$. The subsequent aging starts from a short-time plateau, which is most clearly visible for the upjump data points.

Consider a temperature jump initiated at $t=0$, which is studied by monitoring the subsequent time development of $X$. 

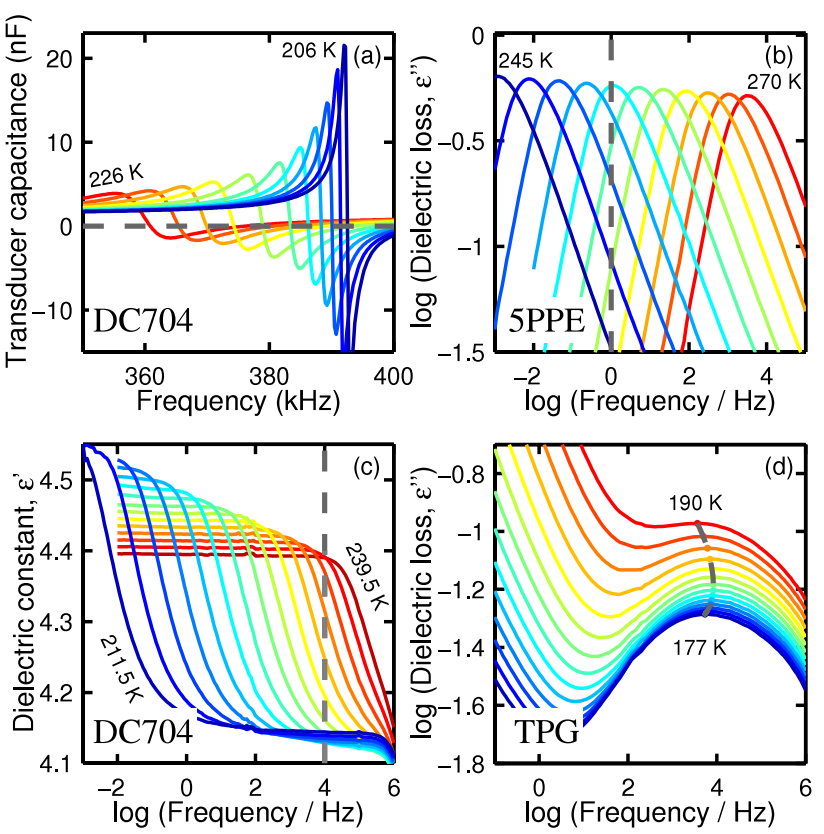

FIG. 1. Spectra illustrating the different types of measurements used for probing physical aging. The intersections between the grey dashed lines and the spectra show the quantities monitored. (a) Mechanical resonance of a piezo-electric disc; (b) dielectric loss; (c) real part of the dielectric constant at a high frequency, which at the aging temperatures is much above the alpha relaxation frequency; and (d) dielectric beta relaxation loss-peak frequency (raw loss-peak frequency with no correction for the alpha process.

The jump starts from equilibrium at temperature $T_{0}+\Delta T$ and ends in equilibrium at $T_{0}$ at which the equilibrium value of $X$ is denoted by $X_{\text {eq. }}$. Following the convention of the aging literature, the time-dependent variation of $X$ after the jump is denoted by $\Delta X(t) \equiv X(t)-X_{\text {eq }}$. Thus, $\Delta X(t)$ goes from $\Delta X(0)$ to zero as $t \rightarrow \infty$ and equilibrium at $T_{0}$ is attained.

The material time of the TN formalism, denoted by $\xi$, is defined from the rate $\gamma(t)$ of the system's "inner clock" as follows:

$$
d \xi=\gamma(t) d t .
$$

The TN formalism implies that for the general temperature variation $T_{0}+\Delta T(t)$, the quantity $\Delta X(t)$ can be written as an instantaneous contribution plus a material-time convolution integral, ${ }^{4}$

$$
\Delta X(\xi)=C \Delta T(\xi)-\int_{-\infty}^{\xi} M\left(\xi-\xi^{\prime}\right) \frac{d \Delta T}{d \xi^{\prime}}\left(\xi^{\prime}\right) d \xi^{\prime} .
$$

Here, $\xi=\xi(t)$ is found by integration of Eq. (1). After a jump at $t=0$ from $T_{0}+\Delta T$ to $T_{0}$, it follows from Eq. (2) that $\Delta X(t)$ $=\Delta T(-C+M(\xi))$.

We study jumps small enough that the jump magnitude obeys $\Delta X(0) \propto \Delta T$. In terms of the dimensionless function $\phi(\xi) \equiv(d T / d X)(-C+M(\xi))$, one has $\Delta X(t)=\Delta X(0) \phi(\xi)$ with $\phi(0)=1$. Defining the normalized relaxation function $R(t)$ by

$$
R(t) \equiv \frac{\Delta X(t)}{\Delta X(0)},
$$

for any temperature jump, we thus have

$$
R(t)=\phi(\xi) \text {. }
$$

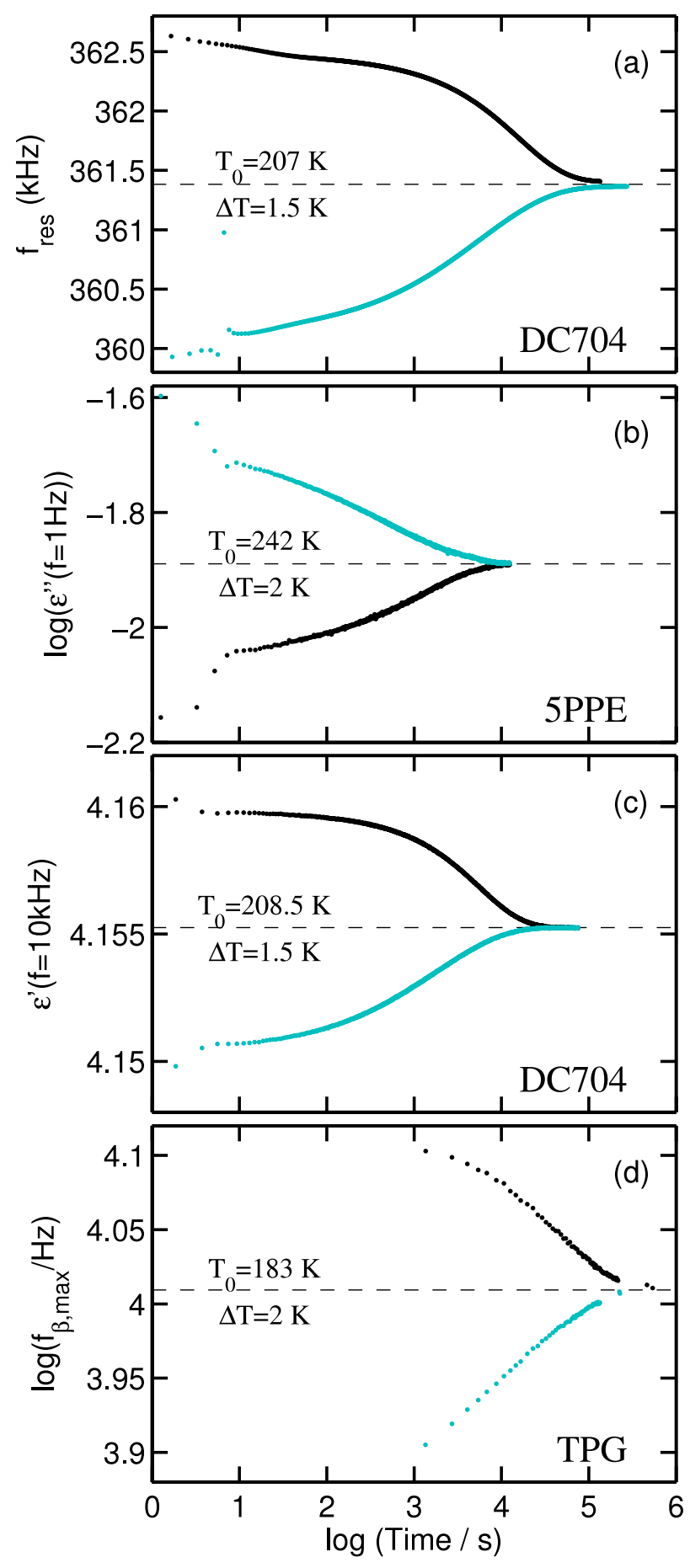

FIG. 2. Aging data for temperature jumps for the different quantities probed. Each panel shows a pair of $\Delta T$ up and down jumps to the same temperature $T_{0}$. Up jumps are shown in black and have the characteristic self-accelerated shape, down jumps in light blue display self-retarded behavior. (a) Mechanical resonance frequency; (b) logarithm of the dielectric loss at $1 \mathrm{~Hz}$; (c) real part of the dielectric constant at $10 \mathrm{kHz}$; and (d) loss-peak frequency of the dielectric beta relaxation.

Having so far followed Narayanaswamy's seminal 1971 paper, ${ }^{4}$ we proceed to convert Eq. (4) into a differential equation. Since $d \xi / d t=\gamma(t)$, the time derivative of $R$ is given by $\dot{R}=\phi^{\prime}(\xi) \gamma(t)$. Equation (4) implies that $\xi$ is a unique function of $R$; thus, $\phi^{\prime}(\xi)$ is also a unique function of $R$. Denoting this (negative) function by $-F(R)$ leads to

$$
\dot{R}=-F(R) \gamma(t) .
$$


Suppose a single parameter $Q$ controls both $X$ and the clock rate. The physical nature of $Q$ is irrelevant. ${ }^{50,55}$ For small temperature jumps, it is reasonable to assume that one can expand $X$ to first order in $Q: \Delta X \equiv X-X_{\text {eq }}=c_{1}\left(Q-Q_{\text {eq }}\right)$ in which $Q_{\text {eq }}$ is the equilibrium value of $Q$ at $T_{0} .{ }^{50}$ The clock rate is determined by barriers to be overcome and their activation energies, so one likewise expects a first-order expansion of the form $\ln \gamma-\ln \gamma_{\mathrm{eq}}=c_{2}\left(Q-Q_{\mathrm{eq}}\right)$ to apply. Eliminating $Q-Q_{\mathrm{eq}}$ leads to $\ln \gamma=\ln \gamma_{\mathrm{eq}}+a \Delta X / X_{\mathrm{eq}}$ in which $a \equiv c_{2} X_{\mathrm{eq}} / c_{1}$ is a dimensionless constant. Introducing the time dependence explicitly via Eq. (3), we have ${ }^{40,56}$

$$
\gamma(t)=\gamma_{\mathrm{eq}} \exp \left(a \frac{\Delta X(0)}{X_{\mathrm{eq}}} R(t)\right) .
$$

Substituting this into Eq. (5) leads to the differential equation for single-parameter aging following a temperature jump,

$$
\dot{R}=-\gamma_{\mathrm{eq}} F(R) \exp \left(a \frac{\Delta X(0)}{X_{\mathrm{eq}}} R\right) .
$$

The important advance of Narayanaswamy in 1971 was to replace that time's nonlinear aging differential equations by a linear convolution integral. It may seem surprising that we now propose stepping back to a differential equation. ${ }^{57}$ Consistency with the TN formalism is ensured, however, by the fact that Eq. (7) only applies for temperature jumps. In contrast, the aging differential equations of Tool and others of the form $d(X-$ $\left.X_{\text {eq }}(T)\right) / d t=-\left(X-X_{\text {eq }}(T)\right) / \tau(X, T)^{40,43}$ were constructed to describe general temperature histories $T(t)$. Such equations lead to simple exponential relaxation in the linear aging limit $(\Delta T \rightarrow 0)$, which is rarely observed, and they cannot account for the crossover effect. ${ }^{10}$

Before proceeding we note the following.

1. In the limit $\Delta T \rightarrow 0$ Eq. (7) becomes $\dot{R}=-\gamma_{\mathrm{eq}} F(R)$. This describes a small temperature jump for which aging is a linear-response phenomenon and the material time reduces to ordinary time. This differential equation determines the convolution kernel of the general aging equation (2). Thus, linear aging determines the general, nonlinear aging.

2. In the long-time limit one has $R(t) \rightarrow 0, \dot{R}(t) \rightarrow 0$, and $\gamma(t) \rightarrow \gamma_{\text {eq }}$. Equation (7) here also reduces to the linearlimit aging equation, $\dot{R}=-\gamma_{\mathrm{eq}} F(R)$. Clearly, $F(R) \rightarrow 0$ for $R \rightarrow 0$. The generic analytic case is $F(R) \propto R$ for $R \rightarrow 0$, which leads to simple exponential relaxation in the longtime limit. The stretched-exponential relaxation function, on the other hand, which is often used to fit aging data, corresponds to a non-analytic $F(R)$ function.

3. If $F(R)=C R$ for all $R$, Eq. (7) may be rewritten to become a differential equation for $\Delta X(t)$. This is a special case of the above-mentioned Tool-type aging equation.

Equation (7) may be tested without fitting data to analytical functions or knowing $F(R)$. Taking the logarithm of Eq. (7) leads to

$$
\ln \left(-\frac{\dot{R}}{\gamma_{\mathrm{eq}}}\right)-a \frac{\Delta X(0)}{X_{\mathrm{eq}}} R=\ln (F(R)) .
$$

For any temperature jump, the left-hand side is predicted to be a function of $R$ that is independent of the jump magnitude $\Delta X(0)$. This is tested in Fig. 3 by plotting the left-hand side
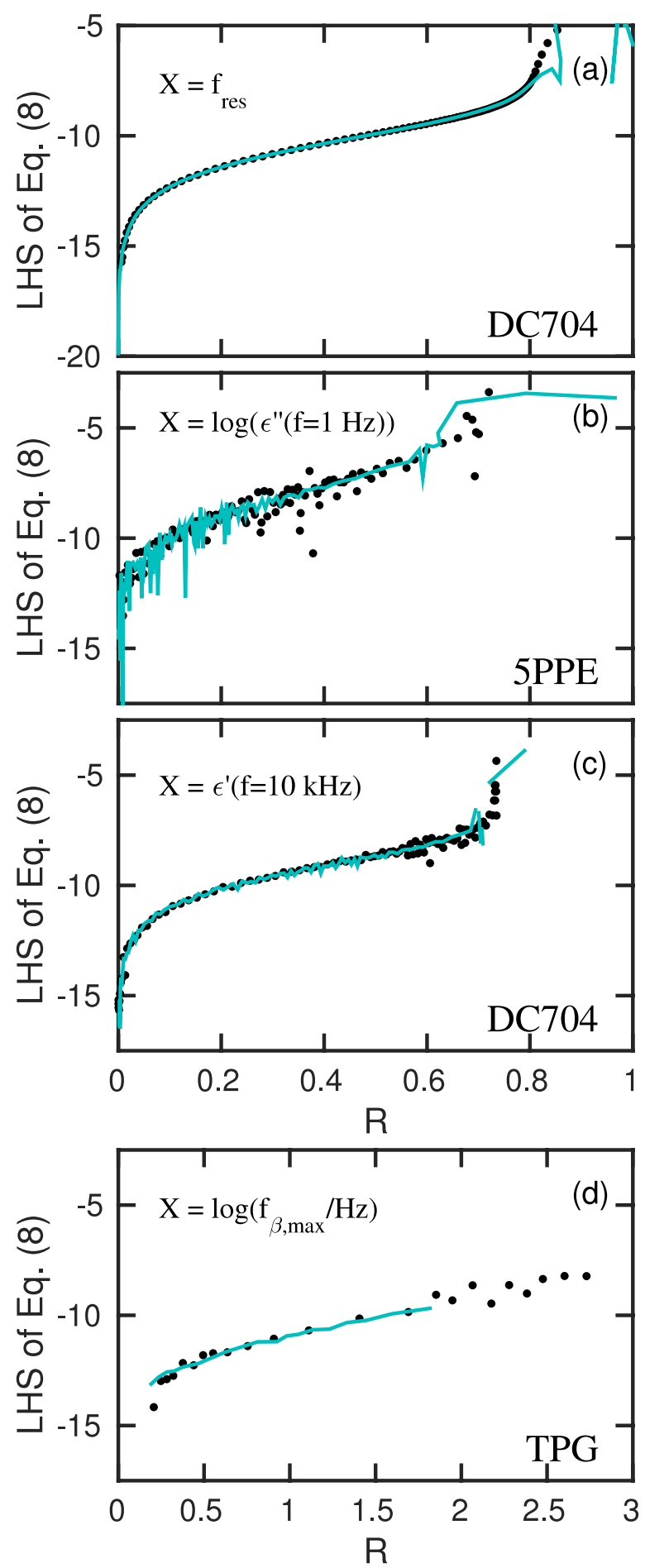

FIG. 3. Test of Eq. (8) for the Fig. 2 data by plotting its left-hand side (except for the factor $\gamma_{\mathrm{eq}}$ ) as a function of the normalized relaxation function $R$ (time unit: seconds; the four $a$ parameters were determined from Eq. (11)). For the first three probes the instantaneous change after a temperature jump goes in the same direction as the subsequent aging. In contrast, the beta loss-peak frequency initially jumps in the opposite direction, which is why $R$ is temporarily larger than unity for this data set (compare Fig. 4(d) below). Data were binned and averaged over ten points, except in (d) where only five points were binned due to scarcity of data. The large black dots give the temperature up jumps; the down jumps are marked by a light blue curve connecting small points.

against $R$ for the data of Fig. 2. The four $a$ parameters have not been optimized for the best fit, but were determined from Eq. (11) derived below. 


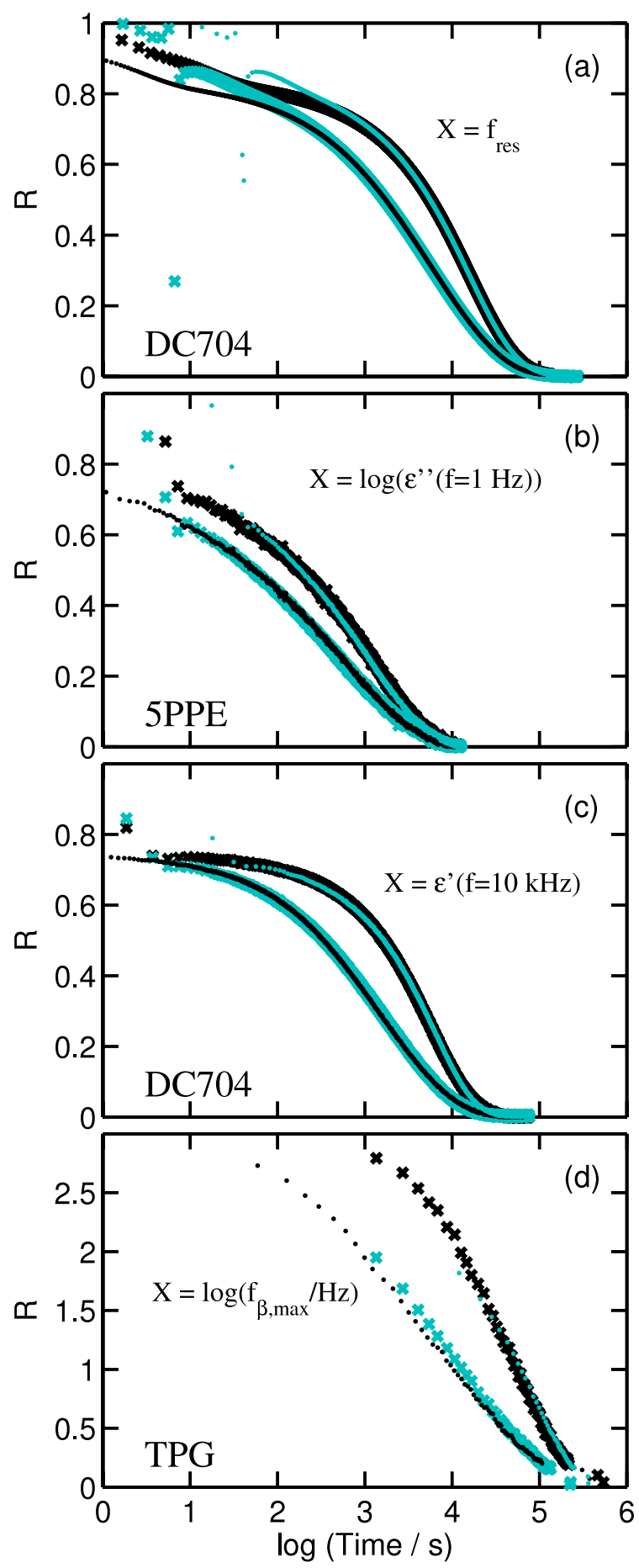

FIG. 4. Data (crosses) and predictions based on Eq. (10) (dots) for each of the normalized Fig. 2 data sets. (a) Mechanical resonance frequency; (b) lowfrequency dielectric loss; (c) high-frequency dielectric constant; and (d) beta loss-peak frequency. Up jumps are in black, down jumps in light blue. The predicted curves are in the same color as the data they are calculated from.

A second test considers two temperature jumps to the same temperature $T_{0}$ (not necessarily of same magnitude). The corresponding normalized relaxation functions are denoted by $R_{1}\left(t_{1}\right)$ and $R_{2}\left(t_{2}\right)$ with inverse functions $t_{1}\left(R_{1}\right)$ and $t_{2}\left(R_{2}\right)$. For times $t_{1}\left(R_{1}\right)$ and $t_{2}\left(R_{2}\right)$ corresponding to the same value of the normalized relaxation functions, $R_{1}=R_{2}$, Eq. (7) implies

$$
\frac{d R_{1}}{d t_{1}} \exp \left(-a \frac{\Delta X_{1}(0)}{X_{\mathrm{eq}}} R_{1}\right)=\frac{d R_{2}}{d t_{2}} \exp \left(-a \frac{\Delta X_{2}(0)}{X_{\mathrm{eq}}} R_{2}\right) .
$$

For time increments $d t_{1}$ and $d t_{2}$ leading to identical changes $d R_{1}=d R_{2}$, if $\Lambda_{12} \equiv a\left(\Delta X_{1}(0)-\Delta X_{2}(0)\right) / X_{\text {eq }}$, Eq. (9) implies $d t_{2}=\exp \left(\Lambda_{12} R_{1}\right) d t_{1}$. By integration and identifying $R \equiv R_{1}$ $=R_{2}$, this leads to

$$
t_{2}(R)=\int_{0}^{t_{2}(R)} d t_{2}=\int_{0}^{t_{1}(R)} e^{\Lambda_{12} R_{1}\left(t_{1}\right)} d t_{1} .
$$

This gives a simple recipe for calculating one normalized relaxation function from another. Figure 4 shows the normalized relaxation functions $R(t)$ of the Fig. 2 data (crosses) and those calculated from the other data set via Eq. (10) (dots).

Equation (10) implies $t_{2}(R)-t_{1}(R)=\int_{0}^{t_{1}(R)}\left(e^{\Lambda_{12} R_{1}\left(t_{1}\right)}\right.$ - 1) $d t_{1}$. A similar expression applies for $t_{1}(R)-t_{2}(R)$. Since $\Lambda_{21}=-\Lambda_{12}$, adding the long-time limits of these expressions leads to the following consistency requirement:

$$
\int_{0}^{\infty}\left(e^{\Lambda_{12} R_{1}\left(t_{1}\right)}-1\right) d t_{1}+\int_{0}^{\infty}\left(e^{-\Lambda_{12} R_{2}\left(t_{2}\right)}-1\right) d t_{2}=0 .
$$

Since $\Lambda_{12}$ determines $a$, this provides an equation for $a$. The four values thus calculated are those used in Figs. 3 and 4 . The supplementary material ${ }^{54}$ shows that the $a$ parameters derived in this way are consistent with extrapolations from highertemperature equilibrium measurements.

In summary, we have presented accurate data for temperature jumps of organic glasses and derived a simplified version of Narayanswamy's 1971 aging theory that allows for direct data tests. The new tests do not involve any fitting to analytical functions. In Ref. 48, we also proposed a test of the Narayanaswamy theory not involving such fits, but it was more complicated and did not make predictions for how to calculate all temperature jumps from knowledge of a single one. Crucially, Eq. (7) involves both the normalized and the unnormalized relaxation functions, $R(t)$ and $\Delta X(t)=\Delta X(0) R(t)$. This is necessary because a differential equation for only $R(t)$ cannot account for the nonlinearity, whereas a simple differential equation involving only $\Delta X(t)$ cannot lead to nonexponentiality in the linear limit.

There are other approaches to describing physical aging than the standard TN theory. ${ }^{36,57}$ The common "singleparameter" assumption of all simple theories is that the quantity monitored correlates to the clock rate $\gamma$. This is also the main ingredient in the approach of Lunkenheimer et al., which assumes a stretched-exponential aging function with a characteristic inverse relaxation time that ages according to the same stretched exponential. ${ }^{36,58}$

Kristine Niss is thanked for several useful discussions. The center for viscous liquid dynamics "Glass and Time" is sponsored by the Danish National Research Foundation via Grant No. DNRF61.

${ }^{1}$ F. Simon, Z. Anorg. Allg. Chem. 203, 219 (1931).

${ }^{2}$ A. Q. Tool and C. G. Eichlin, J. Am. Ceram. Soc. 14, 276 (1931).

${ }^{3}$ A. J. Kovacs, Fortschr. Hochpolym.-Forsch. 3, 394 (1963).

${ }^{4}$ O. S. Narayanaswamy, J. Am. Ceram. Soc. 54, 491 (1971).

${ }^{5}$ C. T. Moynihan, A. J. Easteal, M. A. DeBolt, and J. Tucker, J. Am. Ceram. Soc. 59, 12 (1976)

${ }^{6}$ C. T. Moynihan, P. B. Macedo, C. J. Montrose, P. K. Gupta, M. A. DeBolt, J. F. Dill, B. E. Dom, P. W. Drake, A. J. Easteal, P. B. Elterman et al., Ann. N. Y. Acad. Sci. 279, 15 (1976).

${ }^{7}$ O. Mazurin, J. Non-Cryst. Solids 25, 129 (1977). 
${ }^{8}$ L. C. E. Struik, Physical Aging in Amorphous Polymers and Other Materials (Elsevier, Amsterdam, 1978).

${ }^{9}$ A. J. Kovacs, J. J. Aklonis, J. M. Hutchinson, and A. R. Ramos, J. Polym. Sci.: Polym. Phys. Ed. 17, 1097 (1979).

${ }^{10} \mathrm{G}$. W. Scherer, Relaxation in Glass and Composites (Wiley, New York, 1986).

${ }^{11}$ I. M. Hodge, J. Non-Cryst. Solids 169, 211 (1994).

${ }^{12}$ C. A. Angell, K. L. Ngai, G. B. McKenna, P. F. McMillan, and S. W. Martin, J. Appl. Phys. 88, 3113 (2000).

${ }^{13}$ J. R. White, C. R. Chim. 9, 1396 (2006).

${ }^{14}$ Y. P. Koh and S. L. Simon, Macromolecules 46, 5815 (2013).

${ }^{15}$ J. L. Sullivan, Compos. Sci. Technol. 39, 207 (1990).

${ }^{16}$ I. M. Hodge, Science 267, 1945 (1995).

${ }^{17}$ J. M. Hutchinson, Prog. Polym. Sci. 20, 703 (1995).

${ }^{18}$ G. M. Odegard and A. Bandyopadhyay, J. Polym. Sci., Part B: Polym. Phys. 49, 1695 (2011).

${ }^{19}$ D. Cangialosi, V. M. Boucher, A. Alegria, and J. Colmenero, Soft Matter 9 , 8619 (2013).

${ }^{20}$ D. Cangialosi, J. Phys.: Condens. Matter 26, 153101 (2014).

${ }^{21}$ J. C. Mauro, Unifying Concepts in Glass Physics VI (Aspen Center for Physics, 2015).

${ }^{22}$ L. Grassia and S. L. Simon, Polymer 53, 3613 (2012).

${ }^{23}$ H. S. Chen, J. Appl. Phys. 49, 3289 (1978).

${ }^{24}$ J. C. Qiao and J. M. Pelletier, J. Mater. Sci. Technol. 30, 523 (2014).

${ }^{25}$ L. Lundgren, P. Svedlindh, P. Nordblad, and O. Beckman, Phys. Rev. Lett. 51, 911 (1983).

${ }^{26}$ L. Berthier and J.-P. Bouchaud, Phys. Rev. B 66, 054404 (2002).

${ }^{27}$ O. Kircher and R. Böhmer, Eur. Phys. J. B 26, 329 (2002).

${ }^{28}$ S. M. Fielding, P. Sollich, and M. E. Cates, J. Rheol. 44, 323 (2000).

${ }^{29}$ G. Foffi, E. Zaccarelli, S. Buldyrev, F. Sciortino, and P. Tartaglia, J. Chem. Phys. 120, 8824 (2004).

${ }^{30}$ S. Spinner and A. Napolitano, J. Res. Natl. Bur. Stand., Sect. A 70A, 147 (1966).

${ }^{31}$ Y. Huang and D. Paul, Polymer 45, 8377 (2004).

${ }^{32}$ N. B. Olsen, J. C. Dyre, and T. Christensen, Phys. Rev. Lett. 81, 1031 (1998).

${ }^{33}$ R. Di Leonardo, T. Scopigno, G. Ruocco, and U. Buontempo, Rev. Sci. Instrum. 75, 2631 (2004).
${ }^{34}$ E. Schlosser and A. Schönhals, Polymer 32, 2135 (1991).

${ }^{35}$ R. L. Leheny and S. R. Nagel, Phys. Rev. B 57, 5154 (1998).

${ }^{36}$ P. Lunkenheimer, R. Wehn, U. Schneider, and A. Loidl, Phys. Rev. Lett. 95, 055702 (2005).

${ }^{37}$ R. Richert, Adv. Chem. Phys. 156, 101 (2015).

${ }^{38}$ B. Ruta, Y. Chushkin, G. Monaco, L. Cipelletti, E. Pineda, P. Bruna, V. M. Giordano, and M. Gonzalez-Silveira, Phys. Rev. Lett. 109, 165701 (2012).

${ }^{39}$ C. Brun, F. Ladieu, D. L'Hote, G. Biroli, and J.-P. Bouchaud, Phys. Rev. Lett. 109, 175702 (2012).

${ }^{40}$ A. Q. Tool, J. Am. Ceram. Soc. 29, 240 (1946).

${ }^{41}$ G. B. McKenna, Y. Leterrier, and C. R. Schultheisz, Polym. Eng. Sci. 35, 403 (1995).

${ }^{42}$ J. C. Mauro, R. J. Loucks, and P. K. Gupta, J. Am. Ceram. Soc. 92, 75 (2009).

${ }^{43}$ H. N. Ritland, J. Am. Ceram. Soc. 39, 403 (1956).

${ }^{44}$ I. L. Hopkins, J. Polym. Sci. 28, 631 (1958).

${ }^{45}$ L. W. Morland and E. H. Lee, Trans. Soc. Rheol. 4, 233 (1960).

${ }^{46}$ E. H. Lee and T. G. Rogers, J. Appl. Mech. 30, 127 (1963).

${ }^{47}$ G. B. McKenna, J. Res. Natl. Inst. Stand. Technol. 99, 169 (1994).

${ }^{48}$ T. Hecksher, N. B. Olsen, K. Niss, and J. C. Dyre, J. Chem. Phys. 133, 174514 (2010).

${ }^{49}$ R. Wehn, P. Lunkenheimer, and A. Loidl, J. Non-Cryst. Solids 353, 3862 (2007).

${ }^{50}$ J. C. Dyre and N. B. Olsen, Phys. Rev. Lett. 91, 155703 (2003).

${ }^{51}$ B. Igarashi, T. Christensen, E. H. Larsen, N. B. Olsen, I. H. Pedersen, T. Rasmussen, and J. C. Dyre, Rev. Sci. Instrum. 79, 045105 (2008).

${ }^{52}$ B. Igarashi, T. Christensen, E. H. Larsen, N. B. Olsen, I. H. Pedersen, T. Rasmussen, and J. C. Dyre, Rev. Sci. Instrum. 79, 045106 (2008).

${ }^{53}$ T. Christensen and N. B. Olsen, Rev. Sci. Instrum. 66, 5019 (1995).

${ }^{54}$ See supplementary material at http://dx.doi.org/10.1063/1.4923000 for details on experiments and data analysis, as well as aging data for more liquids.

${ }^{55}$ N. L. Ellegaard, T. Christensen, P. V. Christiansen, N. B. Olsen, U. R. Pedersen, T. B. Schrøder, and J. C. Dyre, J. Chem. Phys. 126, 074502 (2007).

${ }^{56}$ S. E. B. Petrie, J. Polym. Sci., Part A-2 10, 1255 (1972).

${ }^{57}$ I. Kolvin and E. Bouchbinder, Phys. Rev. E 86, 010501 (2012).

${ }^{58}$ R. Richert, P. Lunkenheimer, S. Kastner, and A. Loidl, J. Phys. Chem. B 117, 12689 (2013). 